\title{
Medication adherence in schizophrenia: patient perspectives and the clinical utility of paliperidone ER
}

\author{
Michael Birnbaum' \\ Zafar Sharif ${ }^{2}$ \\ 'St. Luke's-Roosevelt Hospital Center, \\ I I I Amsterdam Ave, New York, \\ NY, USA; ${ }^{2}$ Columbia University \\ College of Physicians and Surgeons, \\ New York, NY, USA
}

\begin{abstract}
Antipsychotic medications provide the foundation for treatment of acute exacerbations as well as relapse prevention in patients with schizophrenia as demonstrated by rigorous placebo-controlled trials. However, despite their proven effectiveness, poor adherence to prescribed antipsychotic regimens remains the most important driver of suboptimal clinical outcomes in this population. This paper reviews the magnitude of the problem of medication non-adherence in patients with schizophrenia and the various factors that contribute to nonadherence, with particular emphasis on factors related to antipsychotic medications. The profile of the latest atypical antipsychotic, paliperidone extended-release (ER) tablets, is then reviewed and the implications of its unique pharmacokinetic profile for adherence in this patient population are discussed.
\end{abstract}

Keywords: schizophrenia, adherence, paliperidone, pharmacokinetics

\section{Overview of adherence}

Schizophrenia can be a devastating mental illness as it impairs fundamental aspects of human emotion and cognition that are essential for successful living in a complex social environment. The illness results in distressing consequences for afflicted patients, their family members, and others involved in their care and support, as well as society at large in terms of lost productivity and cost of providing treatment, housing, and financial assistance. Over the long term, only a minority of patients are gainfully employed or able to live independently. Any new intervention (or improvement in current interventions) will have the potential to improve our ability to optimally care for those affected.

Currently, antipsychotic medication is the primary treatment modality for the management of acute psychosis as well as for relapse prevention in long term maintenance treatment (Lehman et al 1998). While other forms of therapies such as social skills training, cognitive behavioral therapy, and vocational rehabilitation have important roles in the management of patients with schizophrenia, antipsychotics form the foundation of treatment and enable symptom attenuation or resolution so that the benefits of such therapies can be fully realized. In fact, management of schizophrenia cannot be done without appropriate psychotropic treatment both in hospitalized patients as well as in outpatients.

The critical importance of optimal adherence to prescribed antipsychotic regimens has been repeatedly and convincingly demonstrated in patients with schizophrenia. Adherence increases the likelihood of positive outcomes in all aspects of a patient's life including better symptom control (Duncan et al 1998), reduced risk of relapse and rehospitalization (Ascher-Svanum et al 2006), and improvement in quality of life and social and occupational functioning (Ascher-Svanum et al 2006). Conversely, poor 
adherence results in persistence of symptoms and predisposes the patient to relapse which can contribute to a poorer long-term prognosis (Wyatt 1991). Lieberman et al (1996) conducted a longitudinal study in patients with first episode schizophrenia and found that with each successive relapse, response to antipsychotic therapy declined and symptom resolution was not as robust. Unfortunately, the current state of the effectiveness of our treatment interventions is that a significant minority of patients will relapse despite good adherence with prescribed treatment. In one study of patients who responded to treatment for their first psychotic episode, up to $82 \%$ had at least one relapse within 5 years and $78 \%$ had a second relapse within that time period (Robinson et al 1999). As expected, patients who discontinued their medication were almost 5 times more likely to relapse then patients who continued taking medications. These observations highlight the importance of clinicians employing all strategies available to them to optimize pharmacotherapy and enhance adherence with the dual goals of robust symptom attenuation and minimization of relapse risk.

\section{Magnitude of poor adherence}

The rate of non-adherence in patients with schizophrenia has been reported to be as high as 40\%-50\% (Lacro et al 2002). Weiden et al (1991) reported that $74 \%$ of schizophrenic outpatients who are responsive to medications become noncompliant within 2 years post discharge. Even with the newer antipsychotics that are believed to be associated with fewer side effects and better tolerability, the rates of adherence remain poor (Dolder et al 2002).

One major difficulty in researching and assessing the magnitude of non- or poor adherence in schizophrenia is the lack of consistent and agreed upon definitions of this phenomenon (Velligan et al 2006). For instance, some patients who are considered adherent in one study could be categorized as non-adherent in another depending on where the distinction is made. Adherence cannot be conceptualized as an all-or-none phenomenon as only a minority of patients are either "fully compliant" or "fully non-compliant" (Velligan et al 2006) most patients lie somewhere in the middle and are partially or intermittently compliant. However, it has been demonstrated that even minor deviations from prescribed regimens can be associated with deleterious outcomes. A $20 \%$ reduction in medication adherence was found to predict a 3-point increase in scores on the positive and negative symptoms scores (Docherty et al 2002). Another study reported that a gap in treatment of 10 days or less over a 1 -year period doubled the risk of hospitalization (Weiden et al 2004).
A second limitation in adherence research is that there is no reliable methodology for quantifying adherence behavior. The most common method used to assess adherence is information provided by the patient, which for obvious reasons can be significantly flawed (Velligan et al 2006). Self report requires a direct reliance on the patients' recollection and motivations. Patients may overestimate the extent of adherence to please their doctor or avoid confrontation. Patients may also unintentionally provide inaccurate information due to cognitive impairment resulting in an impaired ability to accurately recall their actions (McGrath et al 1997). In addition, even among these self report studies, methods of collecting data differed significantly, ranging from the use of take home questionnaires and diaries to simply having the physician inquire about adherence at each interaction. Similarly, prescribers' assessments of their patients' compliance behavior can be equally flawed (Byerly et al 2007). They may over- or underestimate a patient's adherence based on the clinical presentation of the patient at the time of the interview. Some studies have used the reports of significant others as a means of assessing patient adherence. This, too, may be unreliable for reasons such as the degree of involvement of the individual with the patient and their particular motivations and understanding of the task.

More direct but still flawed "objective" measures that have been used in various studies include pill count and prescription refill records. Pill counting involves counting the number of pills that remain in a prescription bottle after an interval of time. Refill records rely on the number of prescription refills actually obtained by the patient as a proportion of refills that should have been obtained over a time interval. Both these methods have the limitation that they do not measure whether the pill is actually taken by the patient. Electronic pill cap monitoring has been used to count the number of times a medication bottle is opened versus how many times it should have been opened. Here again, it cannot be assessed whether or not the pill was actually ingested.

Urine and blood samples have been used in several studies to measure drug levels but this, too, is not completely reliable as it can only confirm either total absence of drug, or if the drug is present in the specimen, that it was taken in the recent past (based on the half-life of the drug). Plasma or urine level monitoring therefore cannot provide information on the patient's adherence behavior during the entire interval between the time points of monitoring.

Despite these methodological limitations it has been amply demonstrated in the literature that sub-optimal adherence is a major problem in patients with schizophrenia 
and underscores the importance of identifying factors that influence adherence and the need to develop interventions to improve adherence.

\section{Divers of non-adherence}

Several factors that contribute to poor adherence have been identified in compliance research. Some of these are related to the illness, such as poor insight and cognitive dysfunction, while others are linked to the efficacy and tolerability profiles of the medications as well as the route of administration and dosing frequency. Other factors include co-morbid substance abuse (Dixon 1999) and environmental factors such as cultural issues or lack of trust in the treatment team. Multiple drivers of good adherence have also been noted. These include perceived benefit of treatment, fear of hospitalizations, desire to avoid unpleasant psychotic symptoms (Weiden et al 1994), and desire to please the psychiatrist or other members of the clinical team. A good therapeutic alliance will cultivate an environment for improved adherence (Bebbington 1995). Better efficacy and fewer medication side effects will increase adherence as will simple medication regimens and a proper understanding of illness-associated symptoms and the nature of their response to treatment (Degmecic et al 2007).

Lack of insight is one of the most important contributors to non-adherence (Buckley et al 2007). Lack of insight may range from gross denial of illness and a complete rejection of the specific diagnosis to minimization and rationalization of symptoms and a lack of appreciation that medications are required to treat specific symptoms and to reduce the risk of relapse. A significant number of patients with schizophrenia have very poor or complete absence of insight into their illness; such patients are more likely to demonstrate complete rejection of the need for treatment and be chronically non-compliant (Buckley et al 2007). It is therefore of utmost importance to assess a patient's level of insight on a continuum and not simply as "good" or "poor." Does the patient recognize the presence of a mental illness, the nature of the illness and its associated symptoms, and the need for antipsychotic medication for acute as well as maintenance treatment? Deficits in the ability to recognize the presence of a mental illness and the positive therapeutic effect of antipsychotics would clearly increase the likelihood of poor compliance.

Patients with schizophrenia are known to have impairments in cognition which can interfere with their ability to properly comply with prescribed treatment regimens. Cognitive deficits can contribute to possible confusion and failure to execute the act of pill ingestion even when the patient is willing and motivated to comply. In one study, cognitive function (as compared with age, gender, education level, symptom severity, and attitudes toward medications) was the strongest patient-related predictor of a patient's ability to manage medications (Jeste et al 2003). Cognitive deficits can have even greater negative impact on adherence if medications have to be taken multiple times during the day or multiple medications are prescribed. Greenberg et al (1984) demonstrated that patient compliance was as low as $42 \%$ for medications that required qid dosing and $53 \%$ for medications that require tid dosing compared with $70 \%$ compliance for bid and up to $73 \%$ compliance for medications that allow for a single daily dose. Monotherapy is likely to be less cognitively demanding then polypharmacy, and a reduction in the number of different types of medications will decrease the likelihood that a patient forgets to take their medications or has difficulty differentiating between tablets. Patients with schizophrenia are also less likely to accurately recall whether or not they took the medication as prescribed. This further compromises the accuracy of monitoring of compliance in clinical settings especially if the patient is the only source of information regarding compliance behavior.

Psychotic symptoms including delusions, hallucinations and thought disorder can interfere with a patient's ability to properly adhere to antipsychotic treatment as well. Kamali et al (2006) found that high positive symptoms scores in patients at the time of first episode schizophrenia predicted non-compliance at 6-month follow up.

Medication specific characteristics can also affect the ability and the desire of the patient to comply with treatment. For instance, poor response to antipsychotics has been cited as a common reason for stopping medication in schizophrenic patients (Liu-Seifert et al 2005). Understandably if the medication does not appear to be working, adherence is less likely. Along similar lines, the usual lag time required to titrate some medications up to a therapeutic dose and the delay of days to weeks to onset of therapeutic effect in some patients may contribute to non-adherence as patients may lose patience waiting for a response and stop the medication. This time lag may also make it more difficult for the patient to make a connection between taking medication and symptom resolution or conversely, between medication discontinuation and symptom exacerbation (which also does not immediately or invariably ensue from medication cessation). Intolerable side effects also contribute to poor adherence to treatment. Older generation medications such as haloperidol and chlorpromazine were associated with high rates of extrapyramidal symptoms (EPS) which can be very upsetting and uncomfortable for 
the patient. Akathisia, especially, has been shown to be a major reason for medication discontinuation (Van Putten 1978). Overt movement disorders also make the illness more apparent to others further promoting non-adherence so as to not attract undue attention. Newer generation antipsychotics can lead to metabolic disturbances and weight gain which are also difficult for the patient to accept and can impact negatively on medication adherence. In the Clinical Antipsychotic Trials of Intervention Effectiveness (CATIE) (Lieberman et al 2005), the three side effects that resulted in increased rate of medication discontinuation were weight gain, EPS, and sedation. It is not surprising that some studies have reported that older and newer-generation medications do not differentially affect adherence (Menzin et al 2003). Newer drugs, although less likely to cause EPS, are more likely to cause weight gain and be more sedating than the older high potency agents. Additionally, the efficacy and tolerability profile of medications is only one of several factors that affect compliance.

Along with the aforementioned disease-related and medication-related drivers of non-adherence, there are a number of known potential environmental barriers that a patient may need to overcome in obtaining and complying with medication. Some of these include the high cost of the medications and the difficulty in getting to the pharmacy. Patients may also have to overcome the objection or intrusion of family members. Other factors such as the lack of a good therapeutic alliance with the treatment team and co-morbid substance abuse have also been demonstrated to be of significant importance in influencing compliance (Lacro et al 2002).

In summary, several important factors have been identified that contribute to non-adherence and multiple factors will have an additive and potentially compounding effect on compliance. The particular combination of factors that determine compliance behavior in any given individual is quite variable and requires careful clinical assessment as a prerequisite for the development of an individualized treatment plan. At least some of these factors may be amenable to modification from external interventions (eg, psycho-education), and some (eg, level of insight) may evolve as the patient gains experience through the course of the illness. Lastly, as stated earlier, it is important to note that deviation from prescribed regimens lies on a continuum from full and complete non-adherence to occasional missed doses. Patients who completely refuse medications may have very different primary drivers for non-adherence (eg, psychotic denial of illness) than patients who occasionally miss doses (eg, mild cognitive deficits in the context of an absent support system) (see Table 1).

The preceding review of adherence is not meant to be complete and exhaustive and the goal was to provide an overview of the phenomenon in which a more detailed discussion of medication-related factors can occur.

\section{Implications of pharmacodynamic and pharmacokinetic profiles of antipsychotics for adherence}

As discussed above, it is a well established fact that characteristics of antipsychotic medications such as efficacy, side effects, and dosing frequency affect adherence. Piette et al (2007) found that patients with schizophrenia taking medications for both schizophrenia and a co-morbid chronic medical illness showed differential adherence to medications for each individual illness. The authors suggested that factors related to the medication themselves and not the limitations imposed by schizophrenia were the main determinants of differential adherence. However, other factors such as differing level of insight into their psychiatric versus medical illnesses and perceived benefit of different medications may also have contributed to the observed differences.

A thorough understanding of how the pharmacodynamic and pharmacokinetic profiles of antipsychotics translate to the observed clinical profiles of the drugs is of fundamental importance for optimal clinical use of these agents. Having this knowledge provides guidance for rational dosing of drugs and facilitates the prevention (or at least early recognition) of clinical situations in which unintended changes in steady-state plasma level of the antipsychotic secondary to drug-drug interactions potentially alter the efficacy or tolerability of the drug. Even minor improvements in the tolerability, effectiveness,

Table I Factors positively and negatively affecting adherence

\begin{tabular}{|c|c|}
\hline Positive & Negative \\
\hline Perceived benefit of treatment & Poor insight \\
\hline Proper psycho-education & $\begin{array}{l}\text { Cognitive dysfunction; psychotic } \\
\text { symptoms }\end{array}$ \\
\hline $\begin{array}{l}\text { Optimal psychopharmacology } \\
\text { (once a day dosing, few adverse } \\
\text { effects, good efficacy) }\end{array}$ & $\begin{array}{l}\text { Side effects (weight gain, sedation, } \\
\text { orthostasis, akathesia) }\end{array}$ \\
\hline Good therapeutic alliance & Poor efficacy \\
\hline Fear of hospitalization & Substance abuse \\
\hline Social support & $\begin{array}{l}\text { Environmental factors (lack of } \\
\text { trust in physicians, high cost of } \\
\text { medication, limited social support) }\end{array}$ \\
\hline
\end{tabular}


and ease of use of antipsychotics have the potential to improve adherence.

From a pharmacodynamic perspective, medications should ideally have much higher affinity for those receptors $\left(\mathrm{eg}, \mathrm{D}_{2}, 5 \mathrm{HT} 2_{\mathrm{A}}\right)$ that are implicated in efficacy compared to those that mediate side effects, such as $\mathrm{H} 1$ (sedation and weight gain) and $\alpha-1$ (orthostasis). Thus, at the desired therapeutic dose such a drug would occupy the specific "efficacy" target receptors and tend to minimally involve other receptors, translating to an improved efficacy/tolerability profile and potentially better adherence. Unfortunately, an agent with such an ideal receptor binding profile currently does not exist. In fact, for some agents (eg, quetiapine, clozapine) the affinity for the $\mathrm{H} 1$ and $\alpha-1$ receptors is substantially higher than for the $\mathrm{D} 2$ receptor and dose titration is required to minimize the risk of sedation and orthostasis, respectively - this need for dose titration for some agents further delays the time to onset of therapeutic effect. One strategy that has been developed to overcome this limitation of mandatory titration for some agents is to deliver a larger dose of the drug over an extended period of time by utilizing extended-release formulations. By avoiding the high peak plasma levels associated with immediate-release formulations it is possible to initiate treatment at a larger dose which is close to or at the therapeutic dose for that drug. This either eliminates the titration phase or substantially reduces its duration. Extended-release formulations also enable drugs with short half-lives to be administered once daily, which simplifies the dosing regimen and has a potential positive impact on patient adherence. Quetiapine SR (slow release) and paliperidone ER (extended release) are examples of extended-release formulations.

In general, antipsychotic drugs are taken orally and after being absorbed in the gut must pass through the liver before reaching the systemic circulation and ultimately their target site of action in the brain. The drug undergoes first-pass metabolism in the liver which results in inactivation of a fraction of the drug. About $5 \%-10 \%$ of Caucasians are poor metabolizers" and have limited activity of the P-450 2D6 isoenzyme pathway. Such patients may end up with unusually high plasma levels on the usual therapeutic dose of a drug (eg, risperidone) that is primarily metabolized by this pathway, resulting in higher rates of adverse events and poor tolerability. This was confirmed in the study of De Leon et al (2005) in which poor metabolizers had a 2-fold higher odds ratio of discontinuing risperidone due to adverse drug reactions compared to normal (extensive) metabolizers. Although a genetic test is now available to identify poor metabolizers, it is quite expensive and not yet in routine clinical use.

Since most antipsychotics are metabolized in the liver there is also the potential risk for drug-drug interactions mediated by induction or inhibition of the P-450 isoenzyme system. Polypharmacy increases the likelihood of drugs interacting at the level of the liver and affecting the blood levels of either the drug itself or other medications that are co-administered. Antipsychotics do not significantly induce or inhibit the P-450 isoenzymes but may be subject to drug-drug interactions if they are co-administered with another drug known to affect the pathway that is relevant in their metabolism. Depending on whether the relevant P-450 enzymes are induced or inhibited, such drug-drug interactions may result in levels of the antipsychotic that are either sub-therapeutic and have a less then optimal therapeutic effect, or are too high and cause unwanted side effects, respectively. Examples of potent inducers of the P-450 3A4 enzyme system include carbamazepine and phenytoin, while common inhibitors include the macrolide antibiotics, some antifungals, and most protease inhibitors. Fluoxetine and paroxetine are potent inhibitors of the 2D6 isoenzyme. Depending on the primary metabolic pathway of the antipsychotic, a dose adjustment may be necessary if a P-450 inducer or inhibitor is co-administered with the antipsychotic. In a study by Deleon et al (2005), concomitant use of 2D6 inhibitors (fluoxetine or paroxetine) and risperidone was found to increase the discontinuation rate due to adverse drug reactions of risperidone from $30 \%$ to $53 \%$.

We will now proceed with a review of the profile of paliperidone ER, the most recent atypical antipsychotic to receive FDA approval, and discuss possible implications of its unique characteristics for improving adherence in patients with schizophrenia. The review of paliperidone ER is relevant in this context because it is the first atypical antipsychotic that is not metabolized by the liver and has a unique delivery system that minimizes peak to trough plasma level fluctuations. It is important to highlight that the following discussion of the potential impact of its unique pharmacokinetic profile on adherence, although logical and rational, is speculative and has yet to be demonstrated. We hope to raise questions for further investigation pertaining to antipsychotic adherence.

\section{Paliperidone ER}

In December 2006, paliperidone ER was approved by the FDA for the treatment of schizophrenia in the United States and has since been approved in several other countries. Paliperidone 
ER was formulated to combine the active metabolite of risperidone (9-OH risperidone) with an osmotic controlledrelease oral delivery system (OROS) that minimizes peak to trough fluctuations. Paliperidone's mechanism of action is believed to result from its high antagonist activity at the $\mathrm{D}_{2}$ and 5-HT2 2 receptors. It also has additional antagonistic affinity for the histaminic $\mathrm{H} 1$ and adrenergic $\alpha-1$ and $\alpha-2$ receptors. It has no affinity for the muscarinic cholinergic receptors.

The osmotic controlled-release formulation enables slow release of paliperidone over a 24-hour period. After a single oral dose, the plasma concentration gradually rises to reach a peak plasma concentration at about 24 hours. Plasma level fluctuation is reduced with a peak to trough fluctuation of only $38 \%$ for a $12 \mathrm{mg}$ dose of paliperidone ER (which is the maximum approved dose), compared with $125 \%$ for a $4 \mathrm{mg}$ dose of risperidone immediate-release formulation (Paliperidone Product Monograph 2007) (Figure 1). A smoother profile of antipsychotic plasma level with limited peak to trough fluctuation has implications for the overall efficacy/tolerability profile of the drug. At any given therapeutic dose (or plasma level), lower peaks can be predicted to be associated with a lower risk of at least those side effects that are dose-related such as sedation, orthostasis, and EPS. The limited plasma level fluctuation with paliperidone ER is also reflected in lesser fluctuation in $\mathrm{D}_{2}$ receptor occupancy in the brain compared with the immediate-release formulation. A dose of $6 \mathrm{mg}$ of paliperidone ER provides a mean $\mathrm{D}_{2}$ receptor occupancy of $64 \%$ at 22 hours post dose (Karlsson et al 2006), which is in the desirable range $(60 \%-80 \%)$ for antipsychotic effect without exceeding the $80 \% \mathrm{D}_{2}$ receptor occupancy threshold associated with adverse motor side effects. This dose can

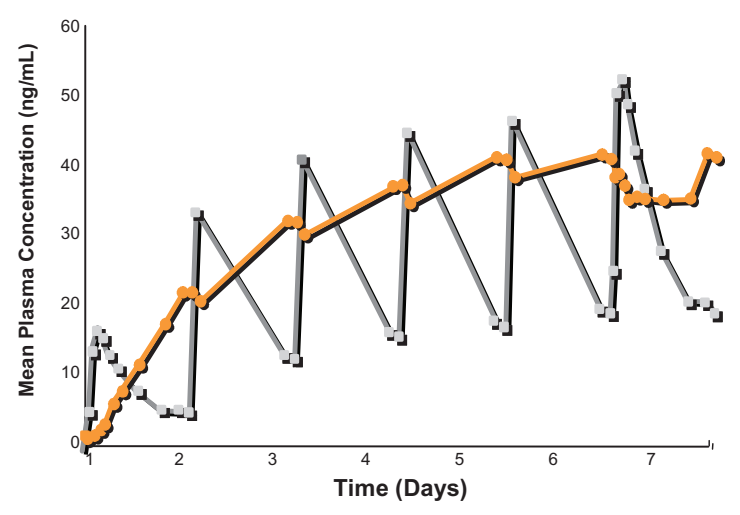

- Paliperidone ER $(n=37) \quad--$ Oral risperidone $(n=37)$

Figure I Plasma concentrations of paliperidone ER ( $12 \mathrm{mg} /$ day) and risperidone $(2 \mathrm{mg}$ first day, then $4 \mathrm{mg} /$ day). Adapted from Eerdekens et al (2006). be administered from Day 1 as the risks of orthostatic hypotension and other unwanted side effects are substantially reduced by the slow release of the medication. Initiating treatment at a therapeutic dose has the added benefit of decreasing the lag time between medication initiation and onset of therapeutic effect. In the clinical trials conducted for regulatory approval, onset of significant therapeutic effect was evident at the first post-baseline assessment point of the studies (Day 4 of treatment). The half-life of paliperidone is approximately 23 hours which allows for once-a-day dosing and results in steady state plasma levels in 4-5 days. The relatively long half-life also limits possible detrimental consequences of occasional missed doses.

Another distinguishing feature of paliperidone ER, other than its delivery system, is that it is the first and only antipsychotic that is not metabolized by the P-450 isoenzyme system, and is only minimally metabolized by other pathways in the liver; approximately $60 \%$ is eliminated unchanged in the urine. Thus, paliperidone would not be expected to be subject to drug-drug interactions mediated through the P-450 system and its dosing would not be expected to differ between slow and rapid metabolizers. The potential for important drug-drug interactions was highlighted in a recently conducted naturalistic analysis across 10 European countries which found that up to $29 \%$ of outpatients with schizophrenia were receiving concomitant anticholinergic medications, up to $23 \%$ were on antidepressants, up to $37 \%$ on anxiolytics, and up to $19 \%$ on mood stabilizers (Haro et al 2006). Such high rates of concomitant therapy dramatically increase the likelihood of clinically significant pharmacokinetic drug-drug interactions with unintended consequences for efficacy or tolerability. In addition, individuals with schizophrenia are known to be at increased risk for infection with HIV, hepatitis C, or both (Cournos et al 2005), as well alcohol abuse (Dixon 1999). These co-morbidities can compromise hepatic function directly thereby affecting metabolism of drugs metabolized in the liver placing patients with schizophrenia at increased risk for adverse drug reactions. Additionally, some of the drugs used to treat these disorders (eg, protease inhibitors) may be inhibitors of some CYP-450 pathways, further complicating treatment by creating potential for drug-drug interactions. The limited hepatic metabolism of paliperidone ER renders it essentially free of the potential for adverse drug-drug interactions; additionally, no dose adjustments are required for patients with mild to moderate hepatic impairment or for those who are slow metabolizers (Paliperidone Prescription Information 2008). This potentially enhances 
the predictability of clinician dosing resulting in improved efficacy/tolerability and therefore improved compliance.

Three randomized, double-blind, placebo-controlled, parallel-group, 6-week trials investigated the efficacy, safety, and tolerability of paliperidone ER at doses of 3-15 $\mathrm{mg}$ per day (Davidson et al 2007; Kane et al 2007; Marder et al 2007). All doses significant reduced schizophrenia symptomatology, with an onset of effect as early as day 4 . The FDA-approved dose range is $3-12 \mathrm{mg}$ /day with a recommended starting dose of $6 \mathrm{mg} /$ day. EPS and weight gain were comparable to placebo in the 3- and 6-mg/day groups but higher than placebo in the 9- and 12-mg/day dose groups. Importantly, the slightly higher rates of EPS in the 9- and 12-mg/day dose groups were not associated with a higher discontinuation rate due to adverse events compared to the placebo group. Prolactin elevation was comparable to that reported in studies with risperidone (Paliperidone Product Monograph 2007).

\section{Conclusion}

Adherence to antipsychotic medication is the single most important determinant of long-term clinical outcome in patients with schizophrenia. Those individuals who are willing and able to fully comply with their treatment regimen will likely maximize their chance of recovery and reduce their risk of relapse over the course of the illness.

A clear understanding by the treatment team of the patient's perspective on their illness and the benefits and risks of antipsychotic treatment (both real and imagined) is the starting point for the formulation of an intervention plan to improve adherence. In any given patient, it is important for clinicians to determine the specific reasons for poor adherence and then tailor treatment and other interventions accordingly. The importance of targeted education about mental illness and the medications used to treat them, with particular focus on the importance of medication adherence and problems that may arise if medications are discontinued, is self evident (Degmecic et al 2007). Providing instructions, or teaching skills that may make medication adherence easier and less demanding is also helpful (Lacro 2006). Improved communication between patients and providers, getting family members involved, and behaviorally focused interventions such as reinforcing specific medication-taking patterns have all been shown to help improve patient adherence. Interventions that include all or most of the aforementioned strategies are typically the most successful (Dolder et al 2003). Intra-muscular injections of depot medications are also available for those patients who are unable or unwilling to comply with oral medications or have repeatedly failed to adhere to daily medication regimens.
For the medications themselves, simplifying medication regimens as much as possible may help patients remain adherent. This makes the task of medication taking less daunting and cognitively taxing. Whenever possible, medications should be administered once daily. It is imperative that clinicians be aware of the pharmacodynamic and pharmacokinetic profiles of the agents they use so as to optimize dosing strategies and recognize situations in which potential drug-drug interactions may occur. Avoiding unnecessary polypharmacy and limiting the number of different medications to the minimum required for control of symptoms will not only make monitoring of clinical response and side effects in patients easier but also help reduce the potential for unintended adverse drug interactions and improve adherence.

The unique pharmacokinetic property of paliperidone ER raises several important questions related to adherence. Does the limited fluctuation in plasma level of the medication improve tolerability and will that translate to improvement in adherence in patients with schizophrenia? Does avoidance of the $\mathrm{P}-450$ system reduce the potential for unwanted drug-drug interactions in real world settings? Is the dosing of paliperidone ER similar for slow versus fast metabolizers and those with hepatic impairment, as would be predicted by its limited hepatic metabolism? Does the limited hepatic metabolism reduce inter-patient variability in plasma level at any given dose secondary to limited first-pass metabolism? If so, does this improve the accuracy of clinician dosing which would in turn improve efficacy and tolerability and possibly adherence? Future research needs to address these important questions.

When choosing among available antipsychotics the important question is not whether one drug is better than the other but, rather, the efficacy attributes and side effect limitations of each drug, and how to dose appropriately each agent based on the diagnosis of the patient, stage of illness, and other factors such as concomitant therapy with potential for drug-drug interactions. This knowledge will surely enable us to make optimal risk benefit judgements when we choose among various available options for individual patients.

\section{Disclosures}

Dr Sharif is on the speakers' bureau for Janssen, Bristol Myers Squibb, and AstraZeneca.

\section{References}

Ascher-Svanum H, Faries DE, Zhu B, et al. 2006. Medication adherence and long-term functional outcomes in the treatment of schizophrenia in usual care. J Clin Psychiatry, 67:453-60.

Bebbington PE. 1995. The content and context of compliance. Int Clin Psychopharmacol, 9(Suppl 5):41-50. 
Buckley PF, Wirshing DA, Bhushan P, et al. 2007. Lack of insight in schizophrenia: impact on treatment adherence. CNS Drugs, 21:129-41.

Byerly MJ, Thompson A, Carmody T, et al. 2007. Validity of electronically monitored medication adherence and conventional adherence measures in schizophrenia. Psychiatr Serv, 58:844-7.

Cournos F, McKinnon K, Sullivan G. 2005. Schizophrenia and comorbid human immunodeficiency virus or hepatitis $\mathrm{C}$ virus. J Clin Psychiatry, 66(Suppl)6:27-33.

Davidson M, Emsley R, Kramer M, et al. 2007. Efficacy, safety and early response of paliperidone extended-release tablets (paliperidone ER): results of a 6-week, randomized, placebo-controlled study.[erratum appears in Schizophr Res. 2007 Nov; 96(1-3):273-4]. Schizophr Res, 93:117-30.

De Leon J, Susce MT, Pan R-M, et al. 2005. The CYP2D6 poor metabolizer phenotype may be associated with risperidone adverse drug reactions and discontinuation. J Clin Psychiatry, 66:15-27.

Degmecic D, Pozgain I, Filakovic P. 2007. Psychoeducation and compliance in the treatment of patients with schizophrenia. Coll Antropol, 31:1111-5.

Dixon L. 1999. Dual diagnosis of substance abuse in schizophrenia: prevalence and impact on outcomes. Schizophr Res, 35(Suppl): S93-100.

Docherty JP, Kozma C, Grogg A, et al. 2002. Antipsychotic maintenance in schizophrenia: Partial compliance and clinical outcome. Am Coll Neuropsychopharmacol, 179.

Dolder CR, Lacro JP, Dunn LB, et al. 2002. Antipsychotic medication adherence: is there a difference between typical and atypical agents? Am J Psychiatry, 159:103-8. [erratum Am J Psychiatry, 159:514].

Dolder CR, Lacro JP, Leckband, et al. 2003. Interventions to improve antipsychotic medication adherence: review of recent literature. J Clin Psychopharmacol, 23:389-99.

Duncan JC, Rogers R. 1998. Medication compliance in patients with chronic schizophrenia: implications for the community management of mentally disordered offenders. J Forensic Sci, 43:1133-7.

Eerdekens E, et al. 2006. US Psychiatric and Mental Health Congress. 16-19 Nov 2006, New Orleans, USA. Poster 335.

Greenberg RN. 1984. Overview of patient compliance with medication dosing: a literature review. Clin Ther, 6:592-9.

Haro JM, Salvador-Carulla L. 2006. The SOHO (Schizophrenia Outpatient Health Outcome) study: implications for the treatment of schizophrenia. CNS Drugs, 20:293-301.

Jeste SD, Patterson TL, Palmer BW, et al. 2003. Cognitive predictors of medication adherence among middle-aged and older outpatients with schizophrenia. Schizophr Res, 63:49-58.

Kamali M, Kelly BD, Clarke M, et al. 2006. A prospective evaluation of adherence to medication in first episode schizophrenia. Eur Psychiatry, 21:29-33.

Kane J, Canas F, Kramer M, et al. 2007. Treatment of schizophrenia with paliperidone extended-release tablets: a 6-week placebo-controlled trial. Schizophr Res, 90:147-61.

Karlsson P, Dencker S, Nyberg S, et al. 2006. Pharmacokinetics and dopamine D2 and Serotonin 5-HT2A receptor occupancy of paliperidone in Healthy Subjects: Two open-label single-dose studies. Clin Pharmacol Ther, 79:74.

Lacro J. 2006. Optimizing adherence to treatment in patients with schizophrenia. Behav Healthc, 26:38-42.
Lacro JP, Dunn LB, Dolder CR, et al. 2002. Prevalence of and risk factors for medication nonadherence in patients with schizophrenia: a comprehensive review of recent literature. J Clin Psychiatry, 63:892-909.

Lehman AF, Steinwachs DM. 1998. Translating research into practice: the Schizophrenia Patient Outcomes Research Team (PORT) treatment recommendations. Schizophr Bull, 24:1-10.

Lieberman JA, Koreen AR, Chakos M, et al. 1996. Factors influencing treatment response and outcome of first-episode schizophrenia: implications for understanding the pathophysiology of schizophrenia. J Clin Psychiatry, 57(Suppl 9):5-9.

Lieberman JA, Stroup TS, McEvoy JP, et al.; and Clinical Antipsychotic Trials of Intervention Effectiveness, I. 2005) Effectiveness of antipsychotic drugs in patients with chronic schizophrenia.[see comment]. N Engl J Med, 353:1209-23.

Liu-Seifert H, Adams DH, Kinon BJ. 2005. Discontinuation of treatment of schizophrenic patients is driven by poor symptom response: a pooled post-hoc analysis of four atypical antipsychotic drugs. $B M C$ Medicine, 3:21.

Marder SR, Kramer M, Ford L, et al. 2007. Efficacy and safety of paliperidone extended-release tablets: results of a 6-week, randomized, placebo-controlled study. Biol Psychiatry, 62:1363-70.

McGrath J, Scheldt S, Welham J, et al. 1997. Performance on tests sensitive to impaired executive ability in schizophrenia, mania and well controls: acute and subacute phases. Schizophr Res, 26:127-37.

Menzin J, Boulanger L, Friedman M, et al. 2003. Treatment adherence associated with conventional and atypical antipsychotics in a large state Medicaid program. Psychiatr Serv, 54:719-23.

Paliperidone (Prescribing Information). 2008. URL: http://www.fda.gov/ cder/foi/label/2006/021999lbl.pdf. Accessed April 42008.

Paliperidone Product Monograph, ER. 2007) http://www.janssen-ortho. com/JOI/pdf_files/Invega_E.pdf. Accessed April 4 2008, page 22.

Piette JD, Heisler M, Ganoczy D, et al. 2007. Differential medication adherence among patients with schizophrenia and comorbid diabetes and hypertension. Psychiatr Serv, 58:207-12.

Robinson D, Woerner MG, Alvir JM, et al. 1999. Predictors of relapse following response from a first episode of schizophrenia or schizoaffective disorder. Arch Gen Psychiatry, 56:241-7.

Van Putten T. 1978. Drug refusal in schizophrenia: causes and prescribing hints. Hosp Community Psychiatry, 29:110-2.

Velligan DI, Lam Y-WF, Glahn DC, et al. 2006. Defining and assessing adherence to oral antipsychotics: a review of the literature. Schizophr Bull, 32:724-42.

Weiden P, Dixon L, Frances A. 1991. Neuroleptic noncompliance in schizophrenia, in Advances in Neuropsychiatry and Psychopharmacology. Schizophr Res, 1:285-96.

Weiden P, Rapkin B, Mott T, et al. 1994. Rating of medication influences (ROMI) scale in schizophrenia. Schizophr Bull, 20:297-310.

Weiden PJ, Kozma C, Grogg A, et al. 2004. Partial compliance and risk of rehospitalization among California Medicaid patients with schizophrenia.[see comment]. Psychiatr Serv, 55:886-91.

Wyatt RJ. 1991. Neuroleptics and the natural course of schizophrenia. Schizophr Bull, 17:325-51. 